\title{
Politische Parteien und Interessengruppen auf der nationalen Ebene und in der EU: umgekehrte demokratische Verhältnisse?
}

\author{
Rinus van Schendelen*
}

\section{Politische Parteien und Interessengruppen in Europa}

Politische Parteien spielen als formale Vertretungsorgane in den Staaten der Europäischen Union (EU) eine entscheidende Rolle für die demokratische Repräsentation. In den 15 „alten“ Staaten übernehmen sie seit etwa einem Jahrhundert die Funktion, die differierenden Interessen der Bürger zu artikulieren, zu aggregieren und in den politischen Willensbildungs- und Entscheidungsprozess einzubinden, kurz: im Namen ihrer Bürger den Staat zu beeinflussen. In den 13 „neuen“ mittel- und osteuropäischen Ländern versuchen sie dies insbesondere nach 1989. ${ }^{1}$ Bürger sehen Parteien jedoch eher als Gebilde an, die ihre gesammelten Wählerstimmen in weitere Machtausübung auf die Regierung umzusetzen versuchen, und weniger als Vertretungsorgane, denen sie handlungsbezogene Repräsentativität zuerkennen. $^{2}$ Interessengruppen sind für Bürger viel ältere Kanäle, um - von innen als Mitglieder oder von außen als Interessenten - gemeinsame Interessen zu entwickeln und damit ihre Außenwelt (Stakeholder im Staat und in der Gesellschaft) zu beeinflussen.

Im Folgenden wird es nicht um die normative Frage gehen, wie diese Kanäle funktionieren sollen ${ }^{3}$, sondern nur um die empirische Frage, ob diese Kanäle auch aktiv genutzt werden. Die zwei Hauptformen gesellschaftlicher Organisationen - Parteien und Interessengruppen - haben eines gemeinsam: das Stichwort „beeinflussen“. Dieses enthält per definitionem nur den Versuch, ein bestimmtes Ziel zu erreichen und nicht das beabsichtigte Endresultat („Einfluss“), das immer auch durch andere Akteure und Faktoren mitbestimmt wird. Etwas „versuchen zu beeinflussen“ ist demnach ein Pleonasmus.

In der Praxis zeigt sich, dass Parteien und Interessengruppen, anders als die Agenda der Massenmedien und der Ministerialbürokratie, in Bezug auf die Interessenvertretung der Wähler beziehungsweise Mitglieder artverwandt sind, da diese Vertretung idealtypisch „von unten nach oben“ (bottom up) verläuft und deshalb zum Thema „Demokratie“ gehört. Getrieben durch ihr Programm oder ihre eigennützige Interessenagenda, konkurrieren sie unter den Bürgern unter anderem um die öffentliche Kommunikation und Meinungsbildung, bei der Werbung um benötigte Unterstützung und Legitimität, um die Artikulation

* Für ihre wertvollen Kommentare zum Konzepttext dankt der Autor vielmals der Redaktion der ZParl sowie Dorothee Mounier-Balduhn, Daniel März und Johannes Pütz.

1 Vgl. David M. Olson / Gabriella Ilonszki (Hrsg.), Special-Issue: Post-Communist Parliaments: Change and Stability in the Second Decade, in: Journal of Legislative Studies, 17. Jg. (2011), H. 2, S. $115-255$.

2 Vgl. Alexander Kühne, Repräsentation enträtselt oder immer noch „the Puzzle of Representation“?, in: ZParl, 44. Jg. (2013), H. 3, S. 459 - 485; Cristina Leston-Bandeira (Hrsg.), Special Issue: Parliaments and Citizens, in: Journal of Legislative Studies, 18. Jg. (2012), H. 3/4, S. 265 - 526.

3 Vgl. Sabine Saurugger, Interest Groups and Democracy in European Union, in: West European Politics, 31. Jg. (2008), H. 6, S. 1274 - 1291; Werner Patzelt, Interessengruppen und Parlamente: Grundsätzliches und Forschungsaufgaben, in: Thomas von Winter / Julia Blumenthal (Hrsg.), Interessengruppen und Parlamente, Wiesbaden 2014, S. 15 - 44. 
und Bildung der gesellschaftlichen Interessen sowie im Staat besonders um die Beeinflussung seiner Willensbildungs- und Entscheidungsprozesse. ${ }^{4}$ Wie die Entwicklung der westlichen Demokratien zeigt, können diese konkurrierenden Aktivitäten eine Partei und/oder Interessengruppe dazu bringen, sowohl formal als auch informell zusammenzuarbeiten beziehungsweise untereinander zu rivalisieren. ${ }^{5}$ Die meisten Parteien sind sogar aus Interessengruppen entstanden, um mittels Wahlen formale Positionen im Staat anzustreben.

Neben diesen gleichgerichteten politischen Aktivitäten gibt es in der Praxis der politischen Systeme auch Unterschiede zwischen Parteien und Interessengruppen. Der wichtigste im vorliegenden Kontext ist der Formalisierungsgrad ihrer Beziehungen zu den Bürgern wie auch zum Staat. Politische Parteien entlehnen ihre Organisationsform dem Wettbewerb durch Parlamentswahlen, die durch das Wahlgesetz und/oder die Verfassung reguliert sind. Regelmäßig müssen sie die Stimmen der Wähler aufs Neue erwerben. Die endgültige Machtposition jeder Partei hängt erstens von ihrem Anteil der erworbenen Stimmen und zweitens von ihrer Koalitionsfähigkeit ab. Die derzeitige Beteiligung an Parlamentswahlen liegt in den meisten EU-Ländern zwischen 60 und 80 Prozent. ${ }^{6}$ Die Parlamente schaffen es jedoch kaum, die zahlreichen differierenden Interessen der Gesellschaft in ihrer Gesamtheit abzubilden. Der soziale Pluralismus wird in den meisten EU-Mitgliedstaaten auf rund zehn verschiedene Parteien im Parlament, das einige hundert Sitze zählt, reduziert. Ihr Organisationsgrad beträgt durchschnittlich 4,7 Prozent der Wahlberechtigten ${ }^{7}$ und in Deutschland 1,8 Prozent (Jahr 2012). ${ }^{8}$

Interessengruppen versuchen hingegen nicht, formale Machtpositionen zu erwerben, sondern informelle Einflusspositionen. Frei von den weitgehenden Restriktionen, die für die Parteien gelten, können sie den sozialen Pluralismus feinmaschiger wiedergeben. Unüberschaubar ist die Zahl der Verbände, die Unternehmen, (Selbst-)Verwaltungseinheiten, Institute und Nichtregierungsorganisationen (NGOs) gebildet haben. Die ihrer jeweiligen Untergliederungen ist um ein Vielfaches höher. Noch viel höher ist die Zahl der Bürger, die als individuelle Mitglieder diesen Einheiten angeschlossen sind, und am allerhöchsten ist die Zahl der Bürger, die die jeweiligen Interessen teilen, zum Beispiel als Arbeitnehmer, Kunde, Einwohner, Sympathisant oder Verbraucher. Kurz, in den EU-Ländern haben weder die Parteien noch die Interessengruppen Kanäle mit festen Anlegestegen bei den Bürgern, doch die der Interessengruppen sind viel zahlreicher und vielgestaltiger als die der Parteien. Auch in anderer Hinsicht sind die Interessengruppen stärker und die Parteien momentan schwächer. ${ }^{9}$ In den legislativen und exekutiven Entscheidungsprozessen haben letztere jedoch stärkere formale Positionen als die Interessengruppen. Zwischen beiden Hauptformen gesellschaftlicher Organisationen gibt es also eine doppelte Inkongruenz.

4 Vgl. Gabriel Almond / G. Bingham Powell, Comparative Politics, Boston 1966.

5 Vgl. Anne Rasmussen / Gert-Jan Lindeboom, Interest Group-Party Linkages in the 21st Century, in: European Journal of Political Research, 52. Jg. (2013), H. 2, S. $264-289$.

6 Vgl. Wolfram Nordsieck, Parties \& Elections. The Database about Parliamentary Elections and Political Parties in Europe, http://www.parties-and-elections.eu (Abruf am 3. September 2014).

7 Vgl. Ingrid van Biezen / Peter Mair / Thomas Poguntke, The Decline of Party-membership in Europe, in: European Journal of Political Research, 51. Jg. (2012), H. 1, S. 24 - 56.

8 Vgl. Oskar Niedermayer, Parteimitgliedschaften im Jahre 2013, in: ZParl, 45. Jg. (2014), H. 2, S. $416-439$.

9 Vgl. Philippe Schmitter, The Changing Politics of Organised Interests, in: West European Politics, 31. Jg. (2008), H. 1/2, S. $195-210$. 
Im Folgenden wird es vor allem darum gehen, wie die nationalen Parteien und Interessengruppen, die seit langer Zeit gemeinsam haben, dass sie ihren Nationalstaat im Namen der Bürger auf je eigene Weise beeinflussen, sich heutzutage auf der EU-Ebene verwandeln, und welche Folgen diese Verwandlungen haben für ihre Fähigkeit, die Politik auf europäischer und nationaler Ebene zu beeinflussen.

\section{Parteien, Interessengruppen und Demokratie}

Kein Fakt ist an sich gut oder schlecht, also auch nicht die festgestellte Inkongruenz zwischen Parteien und Interessengruppen. Nur das gesonderte Denken über eigenständige Werte und Normen kann irgendetwas bewerten und mit Urteilen belegen, die schließlich (inter-)subjektiv sind, und - für den Fall, dass sich eine ernsthafte Diskrepanz zwischen Fakt und Norm zeigt - zu begründeten Maßnahmen führen. So hat es beispielsweise in Bezug auf die heutige formale Machtposition der Parteien im letzten Jahrhundert gute Gründe gegeben, am allgemeinen Wahlrecht als Mechanismus der Demokratie festzuhalten: Erstens sollen bei der periodischen Wahl die Stimmen aller wahlberechtigten Bürger gleiches Gewicht haben, zweitens sollen die Parteien, als Sammler der Wählerstimmen, nach der Wahl eine konstitutionell geschützte Position innehaben. Drittens sollen die Parteien diesen Schutz für die Bildung einer Regierung, die eine Mehrheit repräsentiert, benutzen, und viertens soll diese Regierung stabil sein. Bis heute gehören diese vier Bedingungen zur Essenz des Denkens über Wahldemokratie und sind in allen EU-Ländern kaum umstritten.

Für die schwache Verankerung der Partei in der Gesellschaft gibt es keine guten theoretisch angebbaren Gründe, jedoch gute Erklärungen. Kein politisches System kann sich ständig neuen Wahlen aussetzen, denn das Endprodukt einer stabilen Regierung würde so verfehlt, mit der Folge, dass Parteien, Parteimitglieder und Wähler den Nutzen der Wahldemokratie ernsthaft in Frage stellen würden. Italien, dessen Regierungen seit 1946 im Durchschnitt nur dreizehn Monate überlebten, bildet hierfür ein Beispiel. Der Wahlzyklus beträgt aus guten Gründen in den meisten EU-Staaten regulär vier oder fünf Jahre. Hinzu kommt, dass die meisten Parteien heutzutage nicht über genügend Ressourcen verfügen (zum Beispiel Mitglieder, Organisation und Budget), um die Beziehungen zu den Bürgern in ihrer Rolle als Wähler zu pflegen. Im Wettbewerb miteinander kommunizieren sie mit ihnen durch Massenmedien und inzwischen auch durch die neuen sozialen Medien, die diese Beziehungen weiter anonymisieren.

Im Gegensatz dazu sind die Interessengruppen in ihrer Kommunikation mit den Bürgern nicht durch einen Wahlzyklus oder irgendeine Rolle der Bürger beschränkt. Fortlaufend sprechen sie diese in ihren verschiedenen Rollen (Arbeitnehmer, Einwohner usw.) an und tauschen mit ihnen Informationen und Interessen aus. Sie haben ihnen, die ihre Interessenten sind, vielerlei potenziell relevante Angebote zu bieten. Die Kanäle der Interessengruppen in der Gesellschaft sind nicht gesetzlich durch die Verfassung und/oder das Wahlrecht verbrieft, doch sind sie organisch und/oder organisatorisch aus den eigenen Interessen der Bürger entstanden und demnach vielfach und divers. Ihren Mangel an formalen Machtpositionen auf staatlicher Ebene, wie die Parteien sie erwerben konnten, kompensieren die Interessengruppen dadurch, dass sie Teile des Staates und Parteien, die daran oft großes Interesse haben, beeinflussen. Im Falle der Zusammenarbeit statt Rivalität tauschen 
sie mit ihnen Informationen, Kontakte und Unterstützung aus und versuchen, hierbei Macht und Einfluss zu gewinnen. Ein solcher Austausch findet heute weniger formal und institutionell als vielmehr informell und gelegentlich statt. ${ }^{10}$

Der Frage, ob sowohl Parteien als auch Interessengruppen als intermediäre Organisationen zum Thema „Demokratie“ gehören, sollen drei Vorbemerkungen vorangestellt wer$\operatorname{den}^{11}$ :

(1) Optimierung: In den EU-Ländern ist Demokratie nur ein zentraler Wert neben anderen, wie zum Beispiel gesellschaftliche Integration, effektives und effizientes Verfahren sowie Wohlstand. In der Praxis verursacht diese Vielfalt, dass die meisten Bürger eine Optimierung ihrer Werte der Maximierung eines einzelnen vorziehen. Sie akzeptieren vermutlich auch eine „imperfekte oder defekte" Demokratie, solange diese ihre anderen zentralen Werte fördert.

(2) „Demokratiecontainer“: Innerhalb der EU-Länder bestehen viele Demokratievorstellungen, die aggregiert für die EU-28 eine Art Behälter verschiedener Vorstellungen ergeben, wie in Tabelle 1, gemäß den Phasen (Input, Durchlauf, Output und Feedback) des politischen Prozesses, dargestellt ist. Überall ist die Verwirklichung dieses Containerinhalts stets eine Frage von Optimierung statt Maximierung, da sonst eine Zielvorstellung die andere(n) behindern kann (zum Beispiel kann maximale Transparenz die Entscheidungsfindung behindern und/oder die Rechtsstaatlichkeit beschädigen). Überall löst diese Optimierung einen Streit unter Kritikern um das rechte Maß aus.

(3) Verwandlung: In jedem EU-Mitgliedstaat ist die demokratische Praxis dynamisch statt statisch. In den mittel- und osteuropäischen Ländern kümmern sich viele Bürger heute besonders um die Input- und Feedback-Seite ihres Systems, das vormals relativ geschlossen war, und in vielen südeuropäischen Ländern wünscht man sich mittlerweile einen stärker begrenzten statt dominanten Staat. Auf EU-Ebene, wo die nationalen Vorstellungen von Demokratie (und anderen zentralen Werten) sich begegnen und miteinander konkurrieren, entwickelt sich aus den nationalen Vorstellungen und Praktiken von Demokratie ein neues Amalgam.

Vorläufig kann konstatiert werden, dass in den Nationalstaaten und in der EU sowohl Parteien als auch Interessengruppen an der Verwirklichung vieler Demokratievorstellungen mitarbeiten (können). Sie tun dies auf unterschiedliche Weise und mit je anderen Ergebnissen, setzen auch gelegentlich verschiedene Schwerpunkte. In den Nationalstaaten sind die Parteien oft ein entscheidender Faktor, wenn es etwa um Wahlen, Legitimität und Rechtsstaatlichkeit geht, die Interessengruppen sind ein solcher Faktor hinsichtlich ihrer direkten Kanäle, einvernehmlicher Entscheidungen und Reaktionsfreudigkeit. Ihre Beiträge zur Verwirklichung einiger Demokratievorstellungen sind oft hilfreich, zuweilen notwendig, jedoch selten genügend oder exklusiv. Sowohl die Bürger als auch der Staat, zwischen denen die Parteien und die Interessengruppen als intermediäre Organisationen fungieren, bewirken, dass deren Schwerpunkte und Beiträge variieren und sogar miteinander rivalisieren.

10 Vgl. Anne Rasmussen / Gert-Jan Lindeboom, a.a.O. (Fn. 5).

11 Vgl. Rinus van Schendelen, Die Kunst des EU-Lobbyings. Erfolgreiches Public Affairs Management im Labyrinth Brüssels, Berlin 2012, Kapitel 8. Dieses Buch ist die deutsche Überarbeitung der dritten Ausgabe des Originaltexts „Machiavelli in Brussels: The Art of Lobbying the EU“ (2002), das 2013 als vierte Ausgabe unter dem Titel „The Art of Lobbying the EU: More Machiavelli in Brussels" publiziert ist (Amsterdam University Press). 


\begin{tabular}{|c|c|c|c|}
\hline \multicolumn{4}{|c|}{ Tabelle 1: Populäre Vorstellungen von Demokratie } \\
\hline Input-Vorstellungen & Durchlauf-Vorstellungen & Output-Vorstellungen & Feedback-Vorstellungen \\
\hline $\begin{array}{l}\text { Offenheit, Gleichheit, } \\
\text { Akzeptanz }\end{array}$ & Repräsentation & Legitimität & $\begin{array}{l}\text { Bürgerschaft, } \\
\text { grundlegende } \\
\text { Unterstützung }\end{array}$ \\
\hline $\begin{array}{l}\text { pluralistischer } \\
\text { Wettbewerb }\end{array}$ & $\begin{array}{l}\text { mehrheitliche } \\
\text { Entscheidungen, } \\
\text { einvernehmliche } \\
\text { Entscheidungen, } \\
\text { Polyarchie, Opposition }\end{array}$ & $\begin{array}{l}\text { begrenzte Regierung } \\
\text { beziehungsweise } \\
\text { begrenzter Staat, } \\
\text { Rechtsstaatlichkeit }\end{array}$ & $\begin{array}{l}\text { Toleranz, } \\
\text { Identität }\end{array}$ \\
\hline $\begin{array}{l}\text { Konkurrenzwahlen, } \\
\text { direkte Kanäle, } \\
\text { repräsentative Kanäle }\end{array}$ & $\begin{array}{c}\text { legitime Autorität, } \\
\text { Diskursivität / } \\
\text { abwägende } \\
\text { Betrachtungen, } \\
\text { Transparenz }\end{array}$ & $\begin{array}{l}\text { Rechenschaftspflicht, } \\
\text { Reaktionsfreudigkeit }\end{array}$ & $\begin{array}{l}\text { Freiheiten und Rechte, } \\
\text { Verbindungen } \\
\text { zum Input }\end{array}$ \\
\hline
\end{tabular}

Die Schlussantwort am Ende dieses Aufsatzes wird nicht sein, dass in den einzelnen Ländern und in der EU die Parteien und Interessengruppen immer, sozusagen per definitionem, zum Thema „Demokratie“ gehören. Sie können sich außerhalb der akzeptierten Praktiken und Werte der Demokratie verhalten und werden dann als „schlecht“, „illegitim“ oder, durch die Rechtsprechung, als illegal bewertet. Das individuelle Verhalten darf jedoch niemals für die ganze Kategorie als allgemeingültig gelten. Wenn sowohl Parteien als auch Interessengruppen für die Demokratie als systemrelevant betrachtet werden, kann - abhängig von ihrer Prominenz in einem Land oder zu einer Zeit - von einer Parteiendemokratie (und parlamentarischer Demokratie auf der Parlamentsebene) beziehungsweise Interessengruppendemokratie gesprochen werden. Meistens besteht eine sich stets ändernde $\mathrm{Mi}$ schung der beiden Hauptformen von Demokratie. Die meisten Bürger benutzen beide Hauptformen und der Staat verhält sich zu beiden.

\section{Begriffsbestimmung: Beeinflussung durch Interessengruppen, Lobbyismus und Public Affairs}

In der öffentlichen Meinung der EU-Länder sind viele Verallgemeinerungen in Bezug auf den demokratischen Gehalt der Parteien und Interessengruppen anzutreffen. Sie sind entstanden aus historischen Erfahrungen. In den mittel- und osteuropäischen Ländern halten viele Bürger Parteien für illegitim, um nicht zu sagen für kriminell; sie zeigen mehr Vertrauen in die neuen Interessengruppen als in die Parteien. ${ }^{12}$ In Südeuropa, Frankreich, Italien und Spanien sehen viele Bürger Parteien und Interessengruppen stärker mit dem Staat als mit ihnen verknüpft und vermuten bei ihrem Parlament ein „demokratisches Defizit"13.

12 Vgl. David M. Olson / Gabriella Ilonszki (Hrsg.), a.a.O. (Fn. 1); Tanja Börzel (Hrsg.), Special Issue: Civil Society on the Rise? EU Enlargement and Societal Mobilization in Central and Eastern Europe, in: Acta Politica, 45. Jg. (2010), 1/2, S. 1 - 267.

13 Sophie Meunier-Aitsahalia / George Ross, Democratic Deficit or Democratic Surplus, in: French Politics and Society, 11. Jg. (1993), H. 4, S. $57-69$. 
In Großbritannien wird der starke Wettbewerb von Parteien und Interessengruppen als positiv bewertet. In Deutschland waren die Interessengruppen früher staatlich akzeptiert (seit Mitte des 19. Jahrhunderts) als die politischen Parteien, die in der Zwischenkriegszeit schwach waren, seit 1945 aber stark. Und heute gelten sie, nicht die Interessengruppen, als ein primäres Merkmal der Demokratie. Für ein vorurteilfreies Verstehen der Interessengruppen ist es an dieser Stelle nützlich, ihr Verhalten kurz zu definieren und zu erläutern. ${ }^{14}$

Interessengruppen werden hier definiert als Organisationen und Netzwerke mit Interessen, die sie in ihrer Außenwelt fördern wollen, indem sie relevante Adressaten in Legislative und Exekutive sowie andere Interessengruppen beeinflussen. Es muss betont werden, dass es um zielgerichtetes Beeinflussen geht; ein Sachverhalt, der durch die deutsche Terminologie, in der oft von „Vertretung“ oder „Vermittlung“ die Rede ist, verschleiert wird - gerade so, als ob es nicht (auch) um die eigenen Interessen ginge.

Der Begriff Lobbyismus ist aus der spät-mittelalterlichen „Lobia“ in Italien entstanden, die die Halle oder das Vestibül eines Palastes bezeichnet, wo Bürger sich versammelten, um den Prinzen oder Herren um eine Gunst zu bitten. Heutzutage geht es um einen Raum, wo man sich hinter verschlossenen Türen mit Personen aus Politik, (Ministerial-)Bürokratie oder Gesellschaft verständigen kann. Auf EU-Ebene haben die Kommission (KOM) und das Europäische Parlament (EP) „Lobbyismus“ nicht etymologisch definiert, sondern als pars pro toto jeglicher Beeinflussung, nämlich als „Tätigkeiten, (...), mit denen auf die Politikgestaltung oder Politikumsetzung und die Entscheidungsprozesse der EU-Organe unmittelbar oder mittelbar Einfluss genommen werden soll“ 15 .

In den 1980er Jahren kam auch der amerikanische Begriff Public Affairs Management über Großbritannien, die Niederlande und Schweden nach Europa. Dieser beschäftigt sich nicht angelegentlich, sondern strukturell mit den Bedrohungen und Chancen, die eine Interessengruppe in ihrer Außenwelt (also mit Public Affairs, gleich der lateinischen „res publica") wahrnimmt und, nach gründlicher Analyse, als ihre externe Agenda behandeln (lateinisch „manu agere“, Management), das heißt beeinflussen will. Der Unterschied zum Lobbyismus besteht darin, dass hierbei die meisten Tätigkeiten der Beeinflussung eine methodisch-inhaltliche Vorbereitung („desk research“) erfordern, die gewöhnlich zu höchstens fünfzehn Prozent durch traditionellen Lobbyismus (wie Besuche) zum Erwerb fehlender Informationen und/oder zur Unterstützung durch Schlüsselpersonen ergänzt wird.

Klassischer Lobbyismus bleibt gut möglich in EU-Ländern mit relativ stabiler und konzentrierter Macht bei wenigen Personen innerhalb der Parteipolitik und/oder Bürokratie eine Konstellation, wie sie oft in den älteren südeuropäischen Mitgliedstaaten besteht. In Belgien wird dann ein „Séparée“ besucht, in Frankreich ein „Anti-chambre“, in Italien eine „Lobia“ usw. In den zehn neuen ehemals kommunistischen EU-Ländern musste ab 1989 ein neues politisches System mit zahlreichen neuen Politikern und Beamten aufgebaut werden. Anfänglich glitt der alte Lobbyismus in viele schmutzige Praktiken (Korruption usw.) $\mathrm{ab}$, die im Baltikum, in Polen und der Tschechischen Republik bald zu zahlreichen Bürger-

14 Vgl. Rinus van Schendelen, a.a.O. (Fn. 11), Kapitel 1 und 6.

15 „Vereinbarung zwischen dem Europäischen Parlament und der Europäischen Kommission über die Einrichtung eines Transparenz-Registers für Organisationen und selbstständige Einzelpersonen, die sich mit der Gestaltung und Umsetzung von EU-Politik befassen“ vom 22. Juli 2011, Abs. 8, http://eur-lex.europa.eu/legal-content/DE/TXT/PDF/?uri=CELEX:32011Q0722\%2801 $\% 29 \&$ from=DE (Abruf am 3. September 2014). 
protesten und häufigem Regierungswechsel führten. Dort zeigte sich ab dem Millennium wachsende Aufmerksamkeit für Public Affairs Management. ${ }^{16}$ Die nordwestlichen EUStaaten, aus denen diese Form des Lobbying weiter nach Europa vordrang, erlebten schon in den 1970er Jahren wachsende Instabilität und Machtaufteilung. Deutschland weist im Vergleich zu anderen Ländern relativ stabile und konzentrierte Machtverhältnisse und ausgeprägten Lobbyismus auf, obwohl seine mit dem Public Affairs Management in der EU vertrauten multinationalen Unternehmen und NGOs diese Praktiken immer stärker auch im eigenen Land anwenden. ${ }^{17}$ Die meisten EU-Mitgliedstaaten haben gegenwärtig mit wachsender politischer Instabilität zu tun, was sich unter anderem an verändertem Wahlverhalten und häufiger wechselnden Regierungen zeigt. Auch eine weitere Machtaufteilung ist festzustellen, wie unter anderem an der Verwaltungsdezentralisierung (nach Regionen und Agenturen), ihre vom Staat freieren Marktorganisationen und ihre Europäisierung abzulesen ist. Diese Entwicklungen sind für Public Affairs Management ein fruchtbarer Boden, denn sie machen die methodische Vorbereitung der persönlichen Kontakte durch Schreibtischarbeit notwendig, und Informationssammlung ist heute dank des Internets viel effizienter möglich. Im Kontext dieser gewandelten Bedingungen und Formen der politischen Beeinflussung durch Interessengruppen bedarf es drei weiterer Bemerkungen:

(1) „2E = $M I^{2 “}:$ Wenn die Inhaber politischer Machtpositionen schwierig zu identifizieren und zu behandeln sind, reicht alter Lobbyismus nicht mehr aus. Auf EU-Ebene müssten beispielsweise in jeder Phase einer Gesetzesvorlage dann bald mindestens 28 Lobbybesuche arrangiert werden, was sich weder die Interessengruppe noch die Empfangsseite leisten kann. Wer dennoch auf effektive (zielerreichende) und effiziente (kostengünstige) Weise (abgekürzt: 2E) beeinflussen will, muss sich methodisch darauf vorbereiten. Dies geschieht erstens mittels der Sammlung von vielen Informationen (I) die, analog zur investigativen Arbeit einer Kripo, in Intelligenz $\left(\mathrm{I}^{2}\right)$ umgesetzt werden müssen, und zweitens, da ein Alleingang keine 2E gibt, mittels einer entscheidenden („kritischen“) Interessentenmasse (M).

(2) Die Bürger: Überall betrachten Interessengruppen auch die Bürger immer mehr als ernst zu nehmende Partner, die sie gern in ihre Interessentenmasse (M) aufnehmen. Als Wechselwähler beeinflussen die Bürger heute öfter die politische Stabilität und als Einwohner, Kunden, Mitglieder usw. die Behörden und Marktorganisationen, die eigene Macht erworben haben. Intelligente Interessengruppen und neue NGOs bieten sich an, um sie zu unterstützen. Nur mit klassischem Lobbyismus kann dies unmöglich effektiv und effizient erreicht werden. Hier bietet sich die Methode des Publik Affairs Management an.

(3) Interessenvertretung: Die zweite Bemerkung kann dazu führen, dass der deutsche Euphemismus für Beeinflussung, bislang normativ aufgefasst als Interessenvertretung oder Interessenvermittlung, künftig faktisch bedeutender wird.

16 Vgl. Carla Millar / Peter Köppl (Hrsg.), Special Issue: Public Affairs in Central and Eastern Europe, in: Journal of Public Affairs, 14. Jg. (2014), H. 1, S. 1 - 83.

17 Vgl. Dominik Meier, Interest Representation in Germany, in: Journal of Public Affairs, 14. Jg. (2014), H. 1, S. $22-30$. 


\section{Verhalten der Parteien auf EU-Ebene}

Auf wissenschaftlich methodische Weise festgestellte, auf ihre Zuverlässigkeit und Gültigkeit kontrollierte Fakten ${ }^{18}$ sind unter Hinzufügung eigenständiger Normen notwendige Bedingungen für jede Theoriebildung und ihre Bewertung. Erst danach könnten Maßnahmen folgen, um eine Diskrepanz zwischen Fakten und Werten zu verringern. In den EUMitgliedstaaten existieren hinsichtlich der Parteien und Interessengruppen starke Bewertungen, wobei diese selten auf Fakten beruhen. Die folgende Skizze basiert auf neueren Untersuchungen und beschränkt sich auf Verbindungen nationaler Parteien und Interessengruppen mit der EU. ${ }^{19}$

Durch die national organisierten Direktwahlen für das EP, das neben dem Vorsitzenden (ab den Wahlen 2014) 750 Mitglieder (MdEP) zählt, verteilen die nationalen Parteien die Sitze ihres Landes untereinander. Die so genannte nationale Delegation ist keine Einheit, sondern umfasst alle nationalen Parteien, die im EP mindestens einen Sitz erworben haben. In der EU-28 umfasst das Parlament etwa 200 verschiedene nationale Parteien, die im Durchschnitt weniger als vier Abgeordnete stellen; die deutsche CDU/CSU ist mit 34 Parlamentariern nach der Wahl 2014 die größte national vertretene Partei und zugleich eine kleine Minorität. ${ }^{20} \mathrm{Um}$ die kritische Masse (M) und spezielle EP-Rechte (wie Redezeit, Personal und Budget) zu erreichen, schließen sich die nationalen Parteien zu etwa zehn ziemlich heterogenen politischen Gruppen zusammen, beispielsweise die Europäische Volkspartei (EVP) und die Sozialdemokraten (S\&D). Ihr schon auf nationaler Ebene stark reduzierter Pluralismus, im Vergleich zur gesellschaftlichen Interessenvielfalt, wird hier weiter reduziert erstens zu kleinen Delegationen, zweitens zu wenigen MdEP für jede Partei und drittens zu einer Minoritätsposition innerhalb jeder Gruppe. Eine solche Reduktion ist in jedem multistaatlichen Parlament unvermeidlich der Fall.

Das EP funktioniert ganz anders als jedes nationale Parlament in den EU-Ländern. Anders als diese, die an der Regierungsbildung maßgeblich beteiligt sind, spielte das EP bis zu den Wahlen 2014 in dieser Hinsicht kaum eine Rolle, da die EU über keine vergleichbare Regierung verfügt. Gemäß den EU-Verträgen besteht der Ministerrat aus den nationalen Ministern, deren Regierungen auch ihren jeweiligen Kandidaten für die Kommission vorschlagen, über dessen Ernennung das EP beschränkte Macht hat. Erstmals im Sommer 2014 gelang es der EP-Mehrheit, ihren Kandidaten für den Vorsitz der Kommission (JeanClaude Juncker) beim Europäischen Rat zu erzwingen.

Das EP kann das Verfahren des Rates und der Kommission häufig beeinflussen, was es auf zwei Weisen aktiv und oft effektiv tut.

(1) Resolutionen: Die Parlamentarier produzieren hunderte Resolutionen auf eigene Initiative durch ihre Ausschüsse und ihre so genannten Intergruppen („Interessengruppen der MdEP"). Diese Resolutionen sind formal zwar nicht rechtsverbindlich, stehen jedoch, nach Bestätigung durch das Plenum, meistens nach einiger Zeit auf der Agenda der Kommission. An der Vorbereitung dieser Resolutionen nimmt die Kommission oft informell teil und benutzt dann das EP für die Erweiterung ihrer eigenen Agenda.

18 Vgl. Arnold Brecht, Political Theory, Princeton 1959.

19 Vgl. Rinus van Schendelen, a.a.O. (Fn. 11), Kapitel 2.

20 Vgl. Europäisches Parlament (DG Communication), European Elections 1979 - 2009, Brüssel 2014. Zu den Ergebnissen der Wahl zum Europäischen Parlament 2014 siehe auch den Beitrag von Oskar Niedermayer in diesem Heft der ZParl. 
(2) Gesetzgebung: Das EP nimmt eine teils starke Position bei der Prüfung der jährlich etwa 450 neuen Gesetzentwürfe der Kommission ein (jedoch nicht bei den ungefähr 1.650 Regelungen, die an die Kommission delegiert oder - durch Komitologie - von der Kommission durchgeführt werden, faktisch außerhalb des EP und des Rats bleiben und meistens 80 bis 85 Prozent aller bindenden EU-Beschlüsse ausmachen). ${ }^{21}$ Das EP und der Rat haben beim „ordentlichen Gesetzgebungsverfahren“ (OGV, vormals die so genannte Mitbestimmung) gleiche Macht, jedoch beim „besonderen Gesetzgebungsverfahren“ (BGV, vormals Konsultation genannt) kann das EP nur Empfehlungen abgeben; ein Drittel der Gesetze ist heute dem OGV zuzurechnen, wobei diese Anzahl dank der neuen Verträge wächst. Häufig benutzt das EP seine starke Macht bei Vorlagen im ordentlichen Gesetzgebungsverfahren als Schubkraft, um Vorgänge im BGV zu beeinflussen. Der Rat und zumal der Europäische Rat der EU produzieren viele nicht-verbindliche Resolutionen, meistens adressiert an die Kommission. Daneben ist er mit der Prüfung der Kommissionsentwürfe beschäftigt. In seinem OGV-Wettbewerb mit dem EP, das meistens zu großen Mehrheiten gelangt $^{22}$, ist der Rat oft stark gespalten und somit ein schwacher Akteur, der leicht verliert.

Die Arbeitsweise des EPs ist anders als die der meisten nationalen Parlamente. Seine im Alltagsgeschäft wichtigsten Arbeitsbereiche sind nicht die politischen Gruppen, sondern die etwa 20 ständigen und nach Politikfeldern eingeteilten Ausschüsse mit einer Größe von 50 bis 70 Mitgliedern - also wie ein kleines Nationalparlament in Europa. Mittels der politischen Gruppen wird jede Gesetzesprüfung in die Hände eines federführenden Ausschusses und darin eines Berichterstatters gegeben. Mitberichterstatter aus anderen Ausschüssen und Schattenberichterstatter aus anderen Gruppen innerhalb eines Ausschusses überwachen umgekehrt den Berichterstatter. Dieser muss die Bestätigung seines Berichtes durch die Plenarsitzung beim ordentlichen Gesetzgebungsverfahren und auch die Aufrechterhaltung der Position bei den Verhandlungen mit dem Rat fördern. Dazu benötigt er die in einem reziproken Verhältnis zu ihm stehenden Überwacher aus den anderen Ausschüssen und Gruppen, sowie sie ihn brauchen, um ihre Punkte in seinen Bericht einfließen zu lassen. In diesem Team von etwa zehn MdEP (nebst ihren Mitarbeitern) findet der gesamte gesetzgeberische Willensbildungs- und Aushandlungsprozess eines Gesetzes im EP statt. Die mittlere Dauer dieser Gesetzesarbeit beträgt rund 24 Monate. Während der fünfährigen Wahlperiode ist ein Abgeordneter nur wenige Male Berichterstatter für einen Gesetzentwurf, der meistens einen spezialistisch-technischen Inhalt hat, beispielsweise landwirtschaftliche Buchführungen, die Mikrofinanzierung für Beschäftigung oder die Cadmium enthaltenden Gerätebatterien.

Zwei Gründe sind entscheidend, warum es, anders als auf nationaler Ebene, kaum Raum für ideologische Parteipolitik im EP gibt:

(1) Gelegenheitsplattformen: Jede politische Gruppe besteht aus vielen nationalen Parteien, die trotz ihrer gemeinsamen Fahne beispielsweise der EVP oder der S\&D, oft stark divergierende sachliche Interessen haben. Die Gruppen sind vor allem Gelegenheitsplattformen nationaler Parteien, die sich mit anderen Parteien zusammenschließen, deren ideologisches Profil sie für kompatibler als das der übrigen Parteien halten. Die nationalen Parteien brauchen jedenfalls die stärkere Masse einer Gruppe, um mehr Macht (Rechte, Positionen usw.) erwerben zu können. Da sie noch wenig europäisiert sind, besteht auf 
EU-Ebene bisher keine transnationale Partei mit einer länderübergreifenden Ideologie und Organisation $^{23}$, die eine Gruppe als ihre „Fraktion“ fungieren lassen könnte.

(2) Sachliche Interessenpolitik: Die Arbeitsweise des EPs gibt wenig Raum, um, beispielsweise bei einer Vorlage für landwirtschaftliche Buchführung, viel ideologische Parteipolitik zu betreiben. Jeden Versuch dazu könnten sowohl die Schattenberichterstatter als auch die Vorsitzenden der Gruppen und der Ausschüsse bei der Endprüfung vereiteln. Kurz: Während auf nationaler Ebene als dominante Politikdefinition die ideologische Parteipolitik gilt, ist sie auf europäischer Ebene vielmehr als sachliche Interessenpolitik zu beschreiben. Dies ist auch in den anderen EU-Institutionen der Fall. ${ }^{24}$ Überall entsteht die Mehrheitsbildung aus Verhandlungen und Kompromissen zwischen Personen, die Einfluss oder Macht haben. Sie alle fragen sich im Stillen: „Welchen Nutzen kann meine Partei oder Interessengruppe daraus ziehen, welchen meine Wähler oder Anhänger im jeweiligen Politikfeld?" Dies aber ist die typische Frage einer Interessengruppe.

\section{Verhalten der Interessengruppen auf EU-Ebene}

Auch die nationalen Interessengruppen haben in der EU ein anderes Umfeld als im eigenen Land. Die EU weist eine viel höhere Quantität von Interessengruppen und eine größere Heterogenität unterschiedlicher Interessenlagen auf. Im einwohnerstärksten Land Europas, Deutschland, sind im Jahr 2014 etwa 2.100 Verbände beim Bundestag registriert. Das Gesamtregister von Kommission und Europäischem Parlament (ohne den widerwilligen Rat), die Europäische Transparenz Initiative (ETI), zählt heute etwa 6.200 Interessengruppen, die meisten mit einem ständigen Büro in Brüssel. Sie kommen aus nur wenigen Ländern: ungefähr 60 Prozent aus Deutschland, Großbritannien und Frankreich, 15 Prozent aus den Niederlanden und Belgien und die übrigen 25 Prozent aus den 23 anderen Ländern. ${ }^{25}$ Dazu gibt es viele Interessengruppen aus Nicht-EU-Ländern, wie die USA, die Türkei, usw. Die meisten Interessengruppen Europas haben jedoch kein ständiges Büro und gehen nur zuweilen nach Brüssel und/oder vertrauen auf ihre Europäische Föderation (Dachorganisation). Auch die Verschiedenheit an Interessen ist in Brüssel viel größer. Was in einem Land wichtig ist, spielt oft anderswo kaum eine Rolle. Die höhere Quantität und größere Verschiedenheit haben in Brüssel einen viel stärkeren Wettbewerb zwischen Interessengruppen zur Folge als in jeder nationalen Hauptstadt. Wenn sich beispielsweise in Berlin 15 sehr verschiedene Interessengruppen um eine (polarisierte) Gesetzesvorlage streiten und beim Bundestag 75 Änderungsvorschläge einbringen, sind diese Anzahlen in Brüssel oft um das Zehnfache höher.

23 Vgl. Natalia Timuş / Simon Lightfoot (Hrsg.), Special Issue: Europarties Between „Deepening“ and „Widening“, in: Acta Politica, 49. Jg. (2014), H. 1, S. 1 - 121; Julian Priestly, European Political Parties: The Missing Link, in: Notre Dame Policy Paper 41 vom 22. Oktober 2010, http:// www.notre-europe.eu/media/policypaper_41.pdf?pdf=ok (Abruf am 3. September 2014).

24 Vgl. Björn Lindberg / Anne Rasmussen / Andreas Wartjen (Hrsg.), Special Issue: The Role of Political Parties in the European Union, in: Journal of European Public Policy, 15. Jg. (2008), H. 8, S. $1107-1265$.

25 Vgl. Arndt Wonka / Frank Baumgartner / Christine Mahoney / Joost Berkhout, Measuring the Size and the Scope of the EU Interest Group Population, in: European Union Politics, 11. Jg. (2010), H. 3, S. $463-476$. 
Die EU-Interessengruppen fangen mit ihrer Beeinflussung meistens auf der nationalen Ebene an. Zwar beteiligen sie sich nicht formal an den EP-Wahlen, jedoch beeinflussen viele informell die Kandidatenlisten und Wahlprogramme ihrer befreundeten nationalen Parteien, die nach der EP-Wahl einer politischen Gruppe beitreten. In diesen Gruppen setzen die Interessengruppen ihre informellen Beziehungen fort, zum Beispiel die deutschen Verbände aus Industrie und Landwirtschaft innerhalb der EVP mit den Unionsparteien, die aus Wohlfahrtspflege und Gewerkschaften innerhalb der S\&D mit der SPD und die Interessengruppen aus der Umweltbewegung innerhalb der EP-Grünen mit Bündnis 90/Die Grünen. Sie können sich auch in mehreren anderen EP-Gruppen heimisch fühlen. Der Zutritt zu den Parlamentariern ist einfach, da ihre Kontaktangaben auf der EP-Website stehen. Ein MdEP hat gewöhnlich großes Interesse daran, eine Interessengruppe zu empfangen, da diese nicht mit ideologischen Zielen (die der Abgeordnete schon hat), sondern mit sachlichen Positionen und Praxiserfahrungen, die für eine Vorlage relevant sind, an ihn herantritt. Nach Anhörung weiterer Interessengruppen kann er die erhaltenen nützlichen Informationen und die dann kumulierte Unterstützung nützen $\left(\mathrm{MI}^{2}\right)$, um besser abzuschätzen, was durchführbar ist - genau wie beim Public Affairs Management. Die Interessengruppe ihrerseits erhält eine bessere Chance, ein Ziel zu erreichen beziehungsweise es nicht in Vergessenheit geraten zu lassen (was in der sehr kompetitiven EU schon ein Gewinn ist). Ein solcher Austausch ist der Basismechanismus der Beziehungen zwischen Interessengruppen und Abgeordneten und ist der dort herrschenden Interessenpolitik angepasst, insbesondere dem wechselseitigen Beziehungsmuster und dem zirkulären Austausch zwischen MdEP und Interessengruppen. ${ }^{26}$

Aus nationaler Perspektive gibt es jedoch ein Problem: Die relevanten Berichterstatter sind selten Landsleute. Am Beispiel: 13 Prozent der MdEP sind deutsch, 87 Prozent nicht; die deutsche Landesgruppe ist sogar die größte. Natürlich kann eine Landesgruppe mehr oder weniger Berichterstatterpositionen erwerben, wie es mit 20 Prozent dieser Positionen der deutschen Delegation Mitte 2000 gut gelang. ${ }^{27}$ Dennoch hat jede Interessengruppe eine kleine Chance, auf einen Landsmann als Berichterstatter zu treffen und, da keine mit allen ihren verschiedenen Landsleuten eine enge Vertrauensbeziehung unterhalten kann, ist die effektive Chance noch kleiner. Theoretisch kann die Interessengruppe sich zwar mit einem Berichterstatter einer anderen Nationalität auf Englisch, in der Lingua Franca Europas, austauschen, aber dann ist die Vertrauensbeziehung, die sie für den Austausch braucht, in der Regel schwächer. In der Praxis bitten viele Interessengruppen dann eher, wenn möglich, die Mitberichterstatter und/oder Schattenberichterstatter ihres Landes und/oder ihren Mitarbeiter, als Hilfslobbyist zu agieren. Dilettantische Interessengruppen gehen sogar zu irgendeinem Landsmann, der sehr freundlich und ansprechbar sein kann, jedoch ziemlich irrelevant ist, da er außerhalb des Kernteams steht. Das Problem, dass ihr nationales Umfeld mit dem der EU inkompatibel ist, lösen die professionell arbeitenden Interessengruppen auf dreierlei Weise:

26 Vgl. Daniel März / Johannes Pütz, Vom Lobbyismus zum Public Affairs Management, in: Zeitschrift für Politikberatung, 7. Jg. (2014), H. 1, S. 1 - 14; Kirstin Lindloff/ Stefan Kundolf / Nils Bandelow, Europäisches Parlament und Interessenverbände als Akteure und Adressaten europäischer Verkehrspolitik: Eine interaktionsorientierte Betrachtung, in: Thomas von Winter / Julia von Blumenthal (Hrsg.), a.a.O. (Fn. 3), S. $211-232$.

27 Vgl. Simon Hix / Bjorn Hoyland, The Political System of the EU, London 2011, S. 58. 
(1) Vorphase in der Kommission: Einen Gesetzentwurf beeinflussen sie lieber in dieser Phase, wo unter anderem die Folgenabschätzung, das Blaubuch (die Online-Konsultation), das Grünbuch (die Problemstellung), das Weißbuch (die Lösungsrichtungen) und der Entwurf eines Vorschlags entstehen. Hier können sie etwa 90 Prozent ihrer Interessen absichern, da EP und Rat den verbindlichen Teil eines Vorschlags gewöhnlich nur um zehn Prozent ändern, so dass sie dann dort ihre letzte Chance nutzen können.

(2) EuroFeds: Diese Interessengruppen sind auf EU-Ebene relativ gut organisiert. Sie wissen, dass jede einzelne Interessengruppe eine kritische europäische Masse (M) braucht. Diese erhalten sie durch die Mitgliedschaft ihres Nationalverbandes bei einer ständigen Europäischen Dachorganisation oder Föderation (kurz: EuroFed), wovon es etwa 1.000 gibt, neben noch zahlreicheren befristeten informellen Netzwerken oder Plattformen. Durch diese EuroFeds und/oder Netzwerke ist ihnen eine effektivere und effizientere Einflussnahme möglich. Auch das Vertrauensproblem mit unbekannten Berichterstattern lösen sie dort, etwa indem sie jemanden von einem verbündeten Interessenverband als Hilfsperson einsetzen.

(3) Expertengruppen: Gern beteiligen sich diese an den etwa 2.000 Expertengruppen der Kommission, die Grünbücher, Weißbücher und erste Vorschläge sowohl für Gesetze als auch für untergesetzliche Regelungen entwerfen. Sie existieren, weil die Kommission, als relativ kleinste Bürokratie Europas, nach Unterstützung und Expertise $\left(\mathrm{MI}^{2}\right)$ hungert. Die Generaldirektionen und Dienststellen beschäftigen etwa 25.000 fest angestellte Mitarbeiter, von denen 14.000 Personen mit inhaltlicher Politik beschäftigt sind; eine Stabsabteilung hat weniger als 20 Mitarbeiter.

Diese Expertengruppen sind - besser als die alten Lobias - die spartanisch organisierten Vorportale der Kommission. ${ }^{28}$ Besonders bei ihrer Vorschlagsarbeit für ein neues Gesetz oder eine neue Regelung befriedigt die Kommission ihren Informationsbedarf durch Einladung („Insourcing“) vieler stark rivalisierender Interessengruppen in eine Expertengruppe. Damit erhält sie die bestmögliche Kenntnis über die Regelungsmöglichkeiten des Sachverhalts und deren Resonanz $\left(\mathrm{MI}^{2}\right)$ und verhütet ein Interessenkartell. Im Austausch erhalten die Interessengruppen gute Einflusschancen. Zur Förderung ihrer gemeinsamen Positionen lösen (oft: verhandeln) die Interessengruppen viele ihrer Streitpunkte unter sich. Die Kommission rekrutiert die Experten vorzugsweise aus den Europäischen Föderationen, Netzwerken oder Plattformen, die dadurch neben kritischer Masse auch Fachleute für die Kommissionsrunden liefern können. Die ungefähr 100.000 Fachleute vertreten (etwa im Verhältnis 50:50) sowohl öffentliche (zentrale und dezentralisierte) als auch private (wirtschaftliche und NGO-artige) Interessengruppen; nur politische Parteien fehlen. Keine nationale Regierung hat einen vergleichbaren Mechanismus zur Politikvorbereitung. Die Parteien im EP und die Regierungen im Rat wagen es kaum, den in den Gesetzentwürfen festgelegten Erfolgen der Interessengruppen aus dem jeweils eigenen Land entgegenzutreten. Vermuten diese Interessengruppen, dass noch mehr zu gewinnen ist, nehmen sie die zehn Prozent Chance bei EP und Rat wahr, und um die Kommission nicht unnötig zu irritieren, verhalten sie sich dort meistens diplomatisch. 


\section{Die Verwandlung der nationalen Parteien auf EU-Ebene}

Auf der nationalen Ebene der EU-Länder sind die heutigen Parteien meistens schwach in der Gesellschaft verankert und haben dank des Wahlgesetzes und/oder der Verfassung starke Beziehungen zur Regierung. Die Interessengruppen zeigen eine umgekehrte Inkongruenz: viele und diverse Kanäle zu den Bürgern, jedoch formal schwache zur Regierung. Beide versuchen ihre Schwäche zu kompensieren: die Parteien besonders durch die Massenmedien und die Interessengruppen durch die Beeinflussung der Regierung. Erstere sind fixiert auf ihre Hauptstadt, um die sie sich wie Planeten um die Sonne drehen. In Brüssel sind sie kaum anwesend. Ihre transnationale Partei ist eine sehr schwache EuroFed, ein eigenes Brüsseler Büro fehlt fast allen, und im Inland verwenden sie ausgewählte europäische Themen als innenpolitische Streitpunkte. ${ }^{29}$ Die Interessengruppen dagegen richten ihren Blick auf ihre Arbeitsfelder, wo diese auch liegen mögen: irgendwo im Hinterland, in Europa oder in der Welt. Für sie ist die nationale Hauptstadt nur ein Ort unter vielen. Zwischen den beiden Hauptformen gesellschaftlicher Organisationen ist der wichtigste Unterschied hier, dass sich - gemäß ihrer „natürlichen “ Verbindungen mit ihrem Land - die Parteien schwieriger europäisieren als die Interessengruppen.

Auf EU-Ebene sind die Parteien, im Vergleich zu ihrer Landesebene, schwach etabliert. Es gibt kein Grundgesetz und/oder Wahlgesetz, das den Parteien privilegierte Positionen in der EU verbrieft. Die Wahlen sind national, die Verträge bestimmen die Position des EPs und nicht die der Parteien. Die EP-Geschäftsordnung fördert, dass die Parteien ihre nationale Identität in die europäische Identität einer Gruppe umsetzen. Auf EU-Ebene verflüchtigen sich die nationalen Parteien. Die wenigen, die dies verweigern, können zwar eine Gruppe der „Nichtangeschlossenen“ bilden, jedoch bleibt diese ohne Rechte im Parlament, zersplittert und einflussarm. Auch in den Beziehungen des EP mit der Kommission oder dem Rat spielen einzelne Parteien kaum eine Rolle. Dafür ist jede zu klein und somit zu schwach. Das EP und die Gruppen, in denen sie funktionieren, stehen außerhalb der Expertengruppen der Kommission und der Arbeitsgruppen des Rates. Nur in der letzten Phase des ordentlichen Gesetzgebungsverfahrens, in der Schlichtungsprozedur, sitzen leitende Vertreter des EPs (nicht namens einer Gruppe oder Partei) formal mit denen der Kommission und des Rates an einem Tisch (Trialog). Im Übrigen können die Gruppen, Parteien und Abgeordneten im EP immer informell Personen innerhalb anderer EU-Institutionen beeinflussen. Sie tun dies meistens bei befreundeten Landsleuten und zuweilen bei ihrer Ständigen Vertretung. Solange sie sich nicht als Interessengruppe organisieren, sind sie selten erfolgreich. Das bessere Beispiel geben einige gruppenübergreifende Intergruppen, die ohne nationales Profil ein sachliches Gesamtinteresse vertreten.

Der Aktivismus der nationalen Parteien und Politiker im EP ist stark ausgerichtet auf dessen Innenwelt der parlamentarischen Verfahren, Ausschüsse und Gruppen. Die Ergebnisse dieses Aktivismus können nur selten einer nationalen Partei oder einem Politiker zugeschrieben werden und erreichen nahezu anonym das Hinterland. Seit 2007 zeigen die Fraktionen in vielen Ländern einen wachsenden EU-Aktivismus, der besonders durch zwei Faktoren begünstigt wurde: die Krisen nach 2007 und das Subsidiaritätsverfahren. ${ }^{30}$

$29 \mathrm{Vgl}$. Lisa Anders, Wen kümmert schon Europa? Europa als Wahlkampfthema bei der Bundestagswahl 2013, in ZParl, 45. Jg. (2014), H. 1, S. $55-72$.

30 Vgl. Christina Leston-Bandeira (Hrsg.), a.a.O. (Fn. 2); David M. Olson / Gabriella Ilonszki (Hrsg.), a.a.O. (Fn. 1). 
(1) Die Krisen von Banken, Euro, Staatshaushalt, Wirtschaft usw. haben die Einstellung vieler Bürger zur EU von meistens positiv-gleichgültig in kritisch oder teilweise sogar EUskeptisch verändert. Dies hat neuen Europa-skeptischen Parteien wie etwa der Alternative für Deutschland (AfD) den Boden bereitet. ${ }^{31}$ Auf diese neue Haltung ${ }^{32}$ konnten die Regierungen nicht adäquat reagieren, da sie fast erlahmten durch ihren Spagat zwischen heimischer Wählerpolitik und ihrer Arbeit im Rat. Diesen Spagat versuchten sie auf zwei Weisen zu lösen: durch geringe Transparenz ihrer Ratsaktivitäten und Ratsdokumente ${ }^{33}$ und (besonders Großbritannien, Deutschland, Österreich, Dänemark und die Niederlande) durch häufigere Nein-Stimmen im Rat. ${ }^{34}$ In vielen EU-Ländern haben die wachsende EuropaSkepsis der Bürger und die „lame duck“-Regierungen die Parlamentsfraktionen zum EUAktivismus angeregt oder geradezu angespornt, wie unter anderem durch ihre zahlreicheren Debatten, Vorbehalte, Ausschussaktivitäten und Berichterstatter in EU-Angelegenheiten deutlich wird.

(2) Dank des Lissabon-Vertrages von 2009 berechtigt das neue Subsidiaritätsverfahren die nationalen Parlamente zum Empfang neuer Gesetzentwürfe der Kommission (nicht der Regelungsvorschläge) und ermächtigt ein Drittel von ihnen in der EU, eine Kommissionsvorlage kurzfristig anzuhalten. ${ }^{35}$ Bislang haben die Parlamente nur eine kleine Stichprobe neuer Vorlagen geprüft und lediglich zwei (aus etwa 1.100) angehalten. Die Prozedur hat jedenfalls die nationalen Parlamente aufmerksamer und aktiver gemacht. ${ }^{36}$ Durch ihre Ausschüsse prüfen sie nun die Subsidiarität insbesondere bei einigen Gesetzentwürfen und bei vielen Vorschlägen zur Übertragung neuer Kompetenzen an die EU. Das bestehende EURecht wird, außer bei regulären Gesetzesänderungen, kaum als Streitpunkt angesehen. ${ }^{37}$ In vielen nationalen Parlamenten zeigen die Fraktionen heute die Ambition, mittels ihrer Machtpositionen und ihres Einflusspotentials bei ihrer Regierung, die nationale EU-Agenda und dadurch auch die EU-Entscheidungen zu beeinflussen. Ihre Regierung ist jedoch

31 Vgl. Hajo Boomgaarden / Andreas Schuck / Matthijs Elenbaas / Claes de Vreeze, Mapping EU Attitudes: Euroscepticism and EU Support, in: European Union Politics, 12. Jg. (2011), H. 2, S. 241 - 266; European Parliament, The Europeans and the Crisis, Eurobarometer 74.1, Brüssel 2010, http://ec.europa.eu/public_opinion/topics/eb741parl_en.pdf (Abruf am 3. September 2014); Cécile Leconte, Understanding Euroscepticism, Basingstoke 2010.

32 Vgl. Mikko Mattila / Tapio Raunio, Drifting Further Apart: National Parties and their Electorates on the EU Dimension, in: West European Politics, 35. Jg. (2012), H. 3, S. 589 - 606.

$33 \mathrm{Vgl}$. COSAC, Developments in EU Procedures and Practices Relevant to Parliamentary Scrutiny, Brüssel 2012.

34 Vgl. Vote Watch, a.a.O. (Fn. 22).

35 Vgl. Aron Buzogdny / Andrej Stuchlik, Subsidiarität und Mitsprache: Nationale Parlamente nach Lissabon, in: ZParl, 43. Jg. (2012), H. 2, S. $340-361$.

36 Vgl. European Commission, Annual Report 2012 on Relations between the Commission and National Parliaments, Brüssel 2013, http://ec.europa.eu/dgs/secretariat_general/relations/relations_other/npo/docs/ar_2012_en.pdf (Abruf am 3. September 2013); Heinrich Schröder, Das novellierte Gesetz über die Zusammenarbeit von Bundesregierung und Deutschen Bundestag in EU Angelegenheiten, in: ZParl, 44. Jg. (2013), H. 4, S. 803 - 812; Erol Külahci (Hrsg.), Europeanisation and Party Politics, Colchester 2012; Katrin Auel, Democratic Accountability and National Parliaments Scrutiny in EU Affairs, in: European Law Review, 13. Jg. (2007), H. 4, S. $487-504$.

37 Vgl. Kyriaki Nanou / Han Dorussen, European Integration and Electoral Democracy, in: European Journal of Political Research, 52. Jg. (2013), H. 1, S. 71 - 93; Jan Karlas, National Parliamentary Control of EU Affairs, in: West European Politics, 35. Jg. (2012), H. 5, S. 1095 - 1113. 
fixiert auf den Rat, die Schlussphase der Gesetzgebung, die nicht den besten Einflussmoment darstellt. Folglich sind die Parteien in den nationalen Parlamenten auf Prozeduren statt auf Prozesse fixiert und erst wenig professionalisiert.

\section{Die Verwandlung der nationalen Interessengruppen aufEU-Ebene}

Wie die nationalen Parteien verflüchtigen sich in der EU auch die nationalen Interessenverbände. Im eigenen Land zuweilen dominant, machen sie in der EU die Erfahrung, immer eine Minorität zu sein, so auch der Bundesverband der Deutschen Industrie (BDI), der Deutsche Gewerkschaftsbund (DGB) und der Bankenverband. Die meisten beschäftigen sich seit Jahren aktiv mit der EU und verstehen es, durch Anschluss an eine EuroFed oder ein Netzwerk, effizientere und effektivere Einflussnahme zu erreichen. Nur ein Neuling in der EU versucht, sich als Einzelgänger zu etablieren, jedoch lernt er dann schnell am Beispiel der anderen. Die meisten Verbände treten einer (oder mehreren) bestehenden EuroFed(s) bei. Andere gruppieren sich um zu einer neuen EuroFed (Plattform oder Netzwerk). Ihre nach Region oder Sektor organisierten Mitglieder folgen ihrem Nationalverband oder nehmen die Ausfahrt zu einer anderen, bestehenden oder neuen EuroFed. Die einzelnen Mitglieder, die gut mit der EU vertraut sind, zum Beispiel große Unternehmen, Städte, Institute oder NGO übernehmen dabei dann oft die Führung. Sie alle verfolgen das Ziel, die EU zu beeinflussen, indem sie mit Stakeholdern erstens eine gemeinsame Agenda entwickeln und zweitens nützliche Positionen außerhalb und innerhalb der EU-Institutionen erwerben. Sogar wenn sie in ihrem Heimatland stark und groß sind, sind sie in einer EuroFed stets eine Minorität. Die EuroFeds (und ihre Varianten) zeigen vier aktuelle Trends ${ }^{38}$ :

(1) Gelegenheitsplattformen: Dies ist heute die bevorzugte Form der Einflussnahme von Interessengruppen einer EuroFed, wenn es um die ihnen wichtigsten Gesetzentwürfe und um ihre zentralen Anliegen geht. Dort arbeiten sie mit Stakeholdern zusammen, die die gleichen Interessen verfolgen und somit eine homogenere Interessenmasse (M) ergeben. Diese Plattformen sind öfter auch sektorenübergreifend, zum Beispiel mit Stakeholdern aus den Sektoren Industrie, Umwelt und Forschung zugleich. Die hohe Quantität und die große Diversität von Interessen in der EU bieten viele Möglichkeiten, um pragmatisch und kreativ eine Gelegenheitsallianz zu bilden, und oftmals honoriert die Kommission sie mit einem privilegierten Zugang.

(2) Direkte Mitgliedschaft: Mehr EuroFeds, zum Beispiel EPEE (Energiesparprodukte) und EFPIA (Chemie), akzeptieren die leitenden Interessengruppen innerhalb eines Landesverbandes heute als direkte Mitglieder, die so eine stärkere Rolle spielen können.

(3) Filialisierung: Die stark organisierten EuroFeds mit direkten Mitgliedern im Innern behandeln die nationalen Verbände häufig als ihre Filialen - zum Vorteil dieser großen Mitglieder und zum Nachteil der kleinen und mittelständischen Unternehmen (KMUs) sowie der nationalen Verbände. Unter dem Druck des EP hat die Kommission eine Besserstellung der KMUs unter anderem bei Subventionsanträgen und dem Zutritt zu ihren Expertengruppen verordnet.

38 Vgl. Rinus van Schendelen, a.a.O. (Fn. 11), Kapitel 3. 
(4) Mehrebenen-Beeinflussung: Immer mehr EuroFeds nutzen den Mehrebenen-Ansatz zur Beeinflussung einer EU-Vorlage. Sie verflechten dabei die internationale, transnationale und innerstaatliche Ebene. ${ }^{39}$

Die vier Tendenzen belegen, dass die EuroFeds ihre Mitglieder kräftig europäisieren. Der erste Trend durchbricht die sektoralen Grenzen zwischen den EuroFeds. Immer mehr einzelne Interessengruppen passen sich dem fast immer sektorenübergreifenden Charakter einer „EU-Dossierarena“ 40 an. Die direkte Mitgliedschaft und die Filialisierung verbinden die nationale und die europäische Ebene enger miteinander. Der vierte Trend belegt einen Beeinflussungsansatz, der die Arenen auf verschiedenen Ebenen professionell integriert.

Zudem wirken sich alle vier auf der Nationalebene aus. Die in der EU erfahrenen Interessengruppen sind auch Protagonisten in den nationalen Hauptstädten. Hier bilden sie häufiger sektorenübergreifende Plattformen. Die direkten Mitglieder einer EuroFed verhalten sich zu ihrem Nationalverband autonomer und behandeln ihn als EU-Filiale und das Hinterland als einen Flecken in Europa, der nur einen Teil ihrer Welt darstellt. Zuhause erfahren sie oft, wie oben erwähnt, wachsende politische Instabilität und Verwaltungsdezentralisierung, was Beeinflussung besonders durch Lobby-Praktiken weniger effizient und effektiv macht. Sie ersetzen diese durch moderne Praktiken des Public Affairs Management, deren Anwendung sie in Brüssel kennengelernt haben.

Meistens sind die tatsächlichen Pioniere zuerst die grenzüberschreitend operierenden einzelnen Interessengruppen, in Deutschland zum Beispiel das Unternehmen Siemens, die NGO Transparency International/DE und das Land Niedersachsen. An zweiter Stelle folgen meistens die Verbände mit einigen leitenden Mitgliedern in einem bestimmten Sektor (Wirtschaft, Institute, Regionen, NGOs usw.). Sie brauchen einige Zeit, um die neuartigen Beeinflussungspraktiken zu verstehen, zu akzeptieren und anzuwenden. Die Nachzügler sind oft die Verbände mit größtenteils kleinen und mittelgroßen Mitgliedschaften (die noch mehr Zeit brauchen), die Untergliederungen der nationalen Regierung (die ihre formale Macht in der EU mit faktischem Einfluss dort verwechseln) und die politischen Parteien (die sich ihres Rückstands jetzt bewusst werden). Der Grad an Professionalität im Public Affairs Management ist nicht konstant, da viele interne und externe Faktoren ihn mitbestimmen. Heutige Nachzügler können morgen Pioniere werden, wie die Gruppe der nördlichen mittel- und osteuropäischen Länder belegt. ${ }^{41}$

Es wird Zeit kosten, die zarten Bemühungen um neuen Praktiken professionell auszurichten. Viele Interessengruppen beauftragen anfänglich ihre Abteilung für Public Relations oder Kommunikation mit Public Affairs Management, was wegen der Unterschiede zwischen diesen Disziplinen oft interne Konflikte, schlechte Ergebnisse und Anfängerfehler hervorruft. ${ }^{42}$ So wird beispielsweise die vorbereitende Recherchearbeit vernachlässigt, Publizität statt Stillschweigen praktiziert, eigenständig statt zusammen mit internen Fachleuten und externen Stakeholdern in Allianzen agiert, mit den eigenen Nachfragen statt mit Ange-

39 Vgl. Rainer Eising, Political Economy of State-Business Relations in Europe, London 2009.

40 Eine Arena ist „die virtuelle Versammlung von Stakeholdern, einschließlich EU-Funktionären, mit ihren zur Debatte stehenden Interessen in Hinblick auf ein spezifisches Dossier oder einen bestimmten Zeitpunkt. Ein Dossier ist zumeist ein Papier oder ein Vorschlag seitens der Kommission, kann jedoch auch eine Angelegenheit sein, die durch eine andere Institution oder die Plattform eines Stakeholders weitergeleitet wurde." Rinus van Schendelen, a.a.O. (Fn. 11), S. 183.

41 Vgl. Carla Millar / Peter Köppl, a.a.O. (Fn. 16).

42 Vgl. Dominik Meier, a.a.O. (Fn. 17). 
boten an die Gegenspieler herangetreten sowie ungeduldig in der Arena agiert. Die Erfahrungen der Interessengruppen in der EU beschleunigen diese Lernprozesse. Besonders gilt dies für die EU-Erfahrung, dass eine proaktive und umsichtige Vorbereitung in Bezug auf Bedrohungen und Chancen sowohl in der EU als auch im eigenen Land immer notwendiger wird. ${ }^{43}$ Ein zunehmend komplexeres und dynamischeres Umfeld erfordert höhere Proaktivität und die wachsende Sozialkontrolle größere Umsicht.

Diese Sozialkontrolle findet in der EU seitens der Wettbewerber statt, etwa durch spezialisierte NGOs (wie die britische „State Watch“ und die deutsche „Lobby Control“) und durch Massenmedien. Seit 1992 (das erste EP-Hearing) betrifft sie besonders die Frage des Demokratiegehalts aller Beeinflussung - gemäß den Maßstäben des Publikums. Seit langem haben Kommission und EP ihrerseits die Frage mit einer im Vergleich zu nationalen Institutionen sehr großen Offenheit und Transparenz für Interessengruppen aller Art, die sie auch gern in ihren Arbeitsprozess einbeziehen, beantwortet. Außerdem gibt die Kommission jährlich mehr als eine Milliarde Euro zur NGO-Förderung aus, und im Jahr 2011 hat das EP die NGO „Finance Watch“ zur Bankenkontrolle initiiert. Auch die stark etablierten Interessengruppen suchen gern die Unterstützung von NGOs, falls diese einen guten Ruf genießen und eine hohe Repräsentativität aufweisen. In den letzten Jahrzehnten sind in der EU allerdings auch viele "falsche“ NGOs entstanden. Sie sehen zwar aus wie Bürgerinitiativen, sind jedoch Produkte insbesondere von Regierungen und Unternehmen, um ihre Interessen schöner zu verpacken. Dank der Europäischen Transparenzinitiative von 2011, die bei der Registrierung jeder Gruppe Fragen nach deren Finanzierung stellt, sind nun auch diese falschen NGOs Gegenstand der Sozialkontrolle geworden, die sie zu größerer Umsicht und Praxismoral anhält. Auf Ebene der meisten Nationalstaaten muss sich diese Umsicht noch als Teil des wachsenden Public Affairs Management entwickeln.

\section{Bewertung der Parteien und Interessengruppen aus Demokratieperspektive}

Sowohl die nationalen Parteien als auch die Interessengruppen nehmen formal und faktisch eine andere Position auf der nationalen als auf der EU-Ebene ein, wo sich ihr nationales Profil, wie gesagt, verflüchtigt. Die Parteien gehen auf in den EP-Gruppen, die Interessengruppen in den EuroFeds, und alle sind in ihrer jeweiligen Gruppe nur kleine Mitglieder unter vielen anderen. Die nationalen Parteien haben kaum neue Wege gefunden, um, außer in ihrem EP-Arbeitsbereich, die EU und damit auch ihr EU-Hinterland zu beeinflussen. Im Vergleich dazu haben sich die Interessengruppen, vor allem dank ihrer kollektiven EuroFeds (und Varianten), ihrer eigenständigen Beeinflussung und ihres begehrten Angebotes von Unterstützung und Expertise $\left(\mathrm{MI}^{2}\right)$ an Kommission und EP (die sie dazu gerne einladen), auffällig aktiver und besser in der EU etabliert. Kurzum: die Parteien befinden sich in weitem Rückstand und werden in der neuen Literatur als „die Opfer“ oder „die Verlierer“ der EUIntegration bezeichnet. ${ }^{44}$ Die Interessengruppen hingegen können, abhängig von den Ergebnissen ihrer Beeinflussung, auch Gewinner sein. Der neue Aktivismus der Parteien in den nationalen Parlamenten könnte ihren Rückstand in der EU in Zukunft verringern.

$43 \mathrm{Vgl}$. Rinus van Schendelen, a.a.O. (Fn. 11), Kapitel 4.

44 Vgl. Mikko Mattila / Tapio Raunio, a.a.O. (Fn. 32); Robert Ladrech, Europeanization and National Politics, Basingstoke 2010. 
Die Frage ist nun, wie die unter EU-Verwandlungsdruck stehenden Parteien und Interessengruppen aus demokratischer Perspektive bewertet werden müssen. Wie oben dargelegt, finden sich in der EU zahlreiche Demokratievorstellungen (Tabelle 1), die an anderer Stelle für die nationale und die EU-Ebene ausgearbeitet und besonders auf die Interessengruppen angewendet worden sind. ${ }^{45}$ Hier muss dreierlei offen bleiben: Erstens, ob die mannigfaltigen Vorstellungen in den EU-Ländern „eins zu eins“ auf die EU anwendbar sind ${ }^{46}$; zweitens die Frage, was die „beste“ Demokratiedefinition und ihre Operationalisierung ist, da diese zu allgemein und bis jetzt ohne eindeutige Antwort ist, drittens, und daraus folgend, ob die EU überhaupt demokratisch ist. Bei dieser Frage variieren die Antworten von „Demokratiedefizit“47 und „Demokratieüberschuss“48 zu „nationalem Demokratiedefizit“ ${ }^{\text {“9 }}$, je nach normativer Vorstellung.

Hier soll es um die pragmatische Frage gehen, ob die Parteien und Interessengruppen auf der nationalen und auf der EU-Ebene zur Verwirklichung mehrerer Demokratievorstellungen positiv beitragen (können). Leider gibt es keine wissenschaftlichen Untersuchungen, in denen die Parteien und Interessengruppen auf beiden Ebenen als unabhängige Variablen und die Bestandteile des „Demokratiecontainers“ als abhängige Variablen empirisch untersucht und verglichen worden sind. Man muss sich behelfen mit vielen verschiedenen Teiluntersuchungen, die insbesondere hinsichtlich der Interessengruppen regelmäßig gesammelt und publiziert werden ${ }^{50}$ und auf denen auch die obigen Beschreibungen basieren.

Diese Teiluntersuchungen zeigen, dass sich im Allgemeinen in ihrem Land die Parteien und Interessengruppen eher mehr als weniger um ihre Beziehungen zu den Bürgern kümmern, sich in ihrem Staat anders verhalten (hinsichtlich Machtausübung beziehungsweise Beeinflussung) und sich in der EU verwandeln. Insbesondere zeigt sich, dass sich die Interessengruppen in der EU durch Umgruppierungen besser als die Parteien etablieren, sogar starke Positionen in der Kommission erhalten und den Bürgern somit interessantere $\mathrm{Zu}$ gangskanäle ins Innere der EU als die Parteien bieten (können). Sowohl die Veränderungen in ihren Heimatländern (mehr politische Instabilität und Machtaufteilung) als auch der EU-Wettbewerb treiben die Interessengruppen zu Bemühungen um eine Verstärkung ihrer gesellschaftlichen Verankerung. ${ }^{51}$ Sie versuchen dies mittels „populärer“ NGOs und/oder größerer eigener Repräsentativität, die auf der nationalen und europäischen Ebene mit der wahlbasierten Repräsentativität konkurriert. ${ }^{52}$ Spezifische Beispiele von positiven Demokratiebeiträgen zeigen besonders die EU-Input-Seite und die EU-Durchlaufseite. Teils unter dem Druck von Interessengruppen zeigen Kommission und EP, mehr als die vergleich-

45 Vgl. Rinus van Schendelen, a.a.O. (Fn. 11), Kapitel 8.

46 Vgl. Simon Hix / Abdul Noury / Gérard Roland, Democratic Politics in the European Parliament, Cambridge 2007.

47 Christopher Lord, Democracy in the European Union, Sheffield 1998.

48 Sophie Meunier-Aitsahalia / George Ross, a.a.O. (Fn. 13).

49 Pippa Norris, Democratic Deficit. Critical Citizens Revisited, Cambridge 2011; Vivien Schmidt, Explaining Democracy in Europe, in: Comparative European Politics, 7. Jg. (2009), H. 3, S. 396 $-407$.

50 Vgl. Rinus van Schendelen, a.a.O. (Fn. 11).

51 Vgl. Nieves Pérez-Borragán / Stijn Smismans, Representativeness in the EU, in: Journal of Common Market Studies, 50. Jg. (2012), H. 3, S. $403-421$.

52 Vgl. Sandra Kröger / Dawid Friedrich (Hrsg.), Special Issue: The Representative Turn in EU Studies, in: Journal of European Public Policy, 20. Jg. (2013), H. 2, S. 155 - 307. 
baren nationalen Institutionen, große Offenheit, pluralistischen Wettbewerb und diskursive Entscheidungsfindung. Keine Regierung setzt so viele Expertengruppen („insourced democracy") wie die Kommission ein, führt so viele öffentliche Konsultationen und Folgenabschätzungen durch und berücksichtigt so viele und verschiedene Interessengruppen. Das EP weist diesbezüglich (leichte) Varianten auf, wie zum Beispiel seine Intergruppen, seine Offenheit für Interessengruppenaktivismus und seine einvernehmliche Arbeitsweise zeigen. Diese Fakten sind gleichwohl keine Produkte der Interessengruppen und/oder der Parteien allein. Die Kommission und das EP haben ihnen die Formen vorgegeben, die sie für ihre Leistungen als positiv erfahren. Ein Beispiel solcher „Gesamtproduktion“ ist der Austausch von substantiellem Inhalt und Unterstützung $\left(\mathrm{MI}^{2}\right)$ seitens der Interessengruppen und der Gewährung besserer Einflusschancen seitens der Kommission und des EP. Auch der immer feinmaschigere Verhaltenskodex für Interessengruppen ist unter dem Druck von kritischen (NGO-artigen) Interessengruppen und von den Verbänden der in Brüssel etablierten Gruppen mit ihren Selbstregulierungen entstanden.

Es gibt in der EU auch viele und starke selbstkorrigierende Demokratiemechanismen (vgl. Tabelle 2), die ohne formale Regelung wirksam sind. Dies ist möglich, weil viele „Sender“ und auch „Empfänger“ der Brüsseler Beeinflussungsprozesse, deren Eigennutz mit einer demokratischer funktionierenden EU gedient ist, die populären Demokratievorstellungen benutzen, um effektiver und effizienter Einfluss zu nehmen. Viele Beispiele finden sich oben und andernorts. ${ }^{53}$ Aus Eigennutz wollen sie sich unter anderem einen guten Ruf erwerben und nicht ins Gerede kommen, was sie zu größerer Umsicht, schärferer Sozialkontrolle und spontaner Korrektur fragwürdiger Praktiken der Beeinflussung treibt. Auch für ihren Wettbewerb untereinander verwenden sie Demokratievorstellungen instrumentell. Besonders die in Brüssel gut und länger etablierten Interessengruppen, die viel zu verlieren haben, verbessern oft spontan die demokratische Qualität der EU.

\begin{tabular}{|c|c|c|c|}
\hline \multicolumn{4}{|c|}{ Tabelle 2: Die selbstkorrigierende EU-Lobbydemokratie } \\
\hline Input-Seite & Durchlauf-Seite & Output-Seite & Feedback-Seite \\
\hline $\begin{array}{c}\text { Beitritt von Gruppen } \\
\text { außerhalb }\end{array}$ & $\begin{array}{c}\text { Eigennütze der } \\
\text { Funktionäre }\end{array}$ & interne Kontrollen & offizielle Interferenzen \\
Öffnungen von innen & beobachtende Gruppen & externe Kontrollen & $\begin{array}{c}\text { Suche nach } \\
\text { Unterstützung }\end{array}$ \\
Bereitstellung von & Vorwegnahme & Vorwegnahme & Protestgruppen \\
Positionen & des Outputs & des Feedbacks & neue Gruppen \\
& & & Reputation \\
\hline
\end{tabular}

In den Umfragen über nationale und europäische Demokratie stehen häufig andere Demokratievorstellungen als die soeben genannten im Mittelpunkt, und nur selten wird das Verhalten der Parteien und Interessengruppen verglichen. Implizit auf Parteien und Interessengruppen hinweisen kann die Legitimität der Output-Seite, das heißt, dass die Entscheidungen wegen ihrer einvernehmlichen und fairen Gestaltung vertrauenswürdig

53 Vgl. Rinus van Schendelen, a.a.O. (Fn. 11), Kapitel 7 und 8. 
und somit akzeptabel sind. ${ }^{54}$ Es gibt wenige Legitimitätsdaten über das Verhalten von Parteien und Interessengruppen, die gültig und vergleichbar sind und die vielen Meinungen objektivieren könnten. Zumeist wird Vertrauen in politische Institutionen auf nationaler und europäischer Ebene erhoben. Eine Umfrage zeigt, dass die Mehrheit der EU-Bürger ihren nationalen Institutionen (und besonders den Parteien) viel mehr als dem EP und der Kommission misstrauen. ${ }^{55}$ Die Deutschen misstrauen mehrheitlich ihren nationalen Institutionen ein bisschen weniger als die Bürger der EU-28. In einer anderen Umfrage zum Vertrauen in das EP und in die nationalen Parlamente genießt ersteres unter Bürgern aus 22 EU-Ländern im Durchschnitt 4.52 Vertrauenspunkte auf einer 10-Punkte Skala (10 = hoch) und jedes nationale Parlament durchschnittlich 4.79 - karge Werte mit hoher Variation (die statistischen Abweichungen betragen 1.09 beziehungsweise 0.53). ${ }^{56}$ In 14 (aus 22) Ländern, insbesondere in den mittel- und osteuropäischen Ländern, zeigen die Bürger mehr Vertrauen in das EP als in ihr nationales Parlament. Die deutschen Vertrauenswerte liegen bei 4.27 (EP) und 4.57 (Bundestag) und sind somit niedriger als beide Durchschnittswerte.

Zur Akzeptanz fehlen valide und vergleichbare Daten, die explizit Parteien und Interessengruppen mit einschließen. Es gibt nur sehr indirekte Messungen, zum Beispiel positive wie „Identität“ und „Bürgerschaft“ und negative wie „Protest“ und „Zynismus“. Die öffentlichen Legitimitätsdebatten sind hinsichtlich der Rolle der Parteien und Interessengruppen also faktisch eine Leerstelle für die empirische Forschung. Ein wenig besser ist die empirische Datenlage hinsichtlich der drei folgenden populären Themen:

(1) Die Transparenz der Beeinflussung: Eine NGO wie „Alter-EU“, die sich vor allem dem Problem der Transparenz widmet, vertritt die Auffassung, dass Transparenz maximiert werden muss $^{57}$, während beispielsweise Rainer Wieland, Mitglied im EP-Präsidium, eine Optimierung bevorzugt, da dieser Wert sonst anderen Zielen, zum Beispiel gute Entscheidungsfindung, schaden würde. In immer mehr EU-Ländern geschieht die Verwaltung der Transparenz mittels eines Registers und eines Verhaltenscodexes für Interessengruppen (jedoch nicht für Parteien). Deutschland legte die erste Regelung in Europa vor (1972), das EP folgte 1997 und mehrere andere EU-Länder ebenfalls, insbesondere die mittel- und osteuropäischen Länder (Litauen, Ungarn und Polen seit 2006). ${ }^{58}$ Die Tendenz ist, dass die Regelungen wie auf der EU-Ebene 2011 (und demnächst erneut) regelmäßig verschärft werden. In der Praxis werden sie jedoch kaum eingehalten, besonders nicht in den mittelund osteuropäischen Ländern. Dank der Sozialkontrolle um die ETI herum, sind sie auf

54 Vgl. Lisa Disch, Toward a Mobilization Concept of Democratic Representation, in: American Political Science Review, 105. Jg. (2011), H. 1, S. $100-114$.

55 Vgl. European Commisson, Europeans in 2014, Special Eurobarometer 415, Brüssel 2014; http://ec.europa.eu/public_opinion/archives/ebs/ebs_415_en.pdf; dies., Public Opinion in the EU, Standard Eurobarometer 80, Brüssel 2013, http://ec.europa.eu/public_opinion/archives/eb/ eb80/eb80_first_en.pdf (jeweils Abruf am 3. September 2014).

56 Vgl. Sara Hobolt, Citizen Satisfaction with EU Democracy, in: Journal of Common Market Studies, 50. Jg. (2012), H. 1, S. 88 - 105; Jordi Muňoz / Mariano Torcal/ Eduard Bonet, Institutional Trust and Multilevel Government in the EU, in: European Union Politics, 12. Jg. (2011), H. 4, S. $551-547$.

57 Vgl. Alter-EU, Bursting the Brussels Bubble, Brüssel 2010, http://www.alter-eu.org/sites/default/ files/documents/bursting-the-brussels-bubble.pdf (Abruf am 3. September 2014).

58 Vgl. Craig Holman / William Luneburg, Lobbying and Transparency, in: Interest Groups and Advocacy, 1. Jg. (2012), H. 1, S. $75-104$. 
EU-Ebene effektiver. ${ }^{59}$ Eine Umfrage in 18 EU-Ländern und auf EU-Ebene unter fast 600 Politikern und hohen Amtspersonen zeigt, dass sie die Transparenz verschiedener Interessenvertreter nirgendwo als perfekt erachten, auf EU-Ebene aber besser als in den meisten Mitgliedsländern. ${ }^{60}$ Durchschnittlich sehen sie das Verhalten von Akademikern, ThinkTanks, Journalisten und Anwälten als am wenigsten transparent an, was auch für Deutschland gilt (hier jedoch die Politikberater anstatt der Akademiker).

(2) Die Gleichheit der Zugangschancen: Dieselbe „Alter-EU“ ist auch hier absolut prinzipientreu und kritisiert, dass in den zweiunddreißig neuen industriebezogenen Expertengruppen der Kommission mehr Fachleute aus der Industrie als aus anderen Sektoren sitzen. ${ }^{61}$ Die Kommission verfolgt jedoch ihr eigenes Interesse, den Zutritt des sehr heterogenen Industriesektors nach dem Gesichtspunkt der breitest möglichen Interessenverschiedenheit der Experten zu regeln. Diese müssen dann im Wettbewerb untereinander die Unverzichtbarkeit ihrer Expertise und Unterstützung demonstrieren (Nachweis von $\mathrm{MI}^{2}$ ). Jedenfalls gewährt die Kommission den Interessengruppen gleichberechtigteren Zugang als die nationalen Regierungen, die sich auf ihre (meistens größeren) Beamtenapparate verlassen und ansonsten ihre parteipolitisch befreundeten Interessengruppen bevorzugen, was in Brüssel bei weitgehender Abwesenheit von Parteipolitik selten geschieht. Die Praxis ist noch komplexer: Kommission und EP begünstigen neue und oft NGO-artige Interessengruppen sowohl mit finanziellen Mitteln als auch mit Positionen in ihren Experten- oder Intergruppen. Bei der Kommission gut etablierte Interessengruppen haben ihrerseits, dank ihres Wettbewerbs untereinander, oft ein großes Interesse daran, nützliche Außenseiter wie NGOs und KMUs herein zu holen. Ohnehin sind Zugang und Einflusserfolg nie identisch. ${ }^{62}$

(3) Das legitime Verhalten: Dass sich Parteien und Interessengruppen innerhalb der Grenzen der Gesetze und Kodizes frei bewegen können, ist prinzipiell nicht umstritten, aber umso mehr sind es diese Grenzen, die Produkte Politischer Kultur sind. Dazu gehören auch neue Auffassungen über „illegitimes Verhalten“. Die Fraktionen haben hier eine komplexe Position: sie haben die Macht, die Grenzen und somit die Verhaltensfreiheit der anderen zu ändern und für sich selber Ausnahmen zu machen, wie es beispielsweise die ETI 2011 für Parteien und nationale Behörden tat. In den öffentlichen Debatten vieler EULänder erzeugt zumal der alte Lobbyismus ein Nebengeräusch, das nach Korruption, Bestechung, Betrug usw. klingt, kurz: nach „illegitimer“ oder sogar „illegaler“ Beeinflussung, die stark kritisiert wird. Das entsprechende Verhalten in den Mitgliedstaaten variiert stark, da die Normen und Formen der Beeinflussung größtenteils in die nationale Kompetenz fallen. Die Kommission publizierte 2014 ihren ersten Anti-Korruptionsbericht ${ }^{63}$, basierend auf

59 Vgl. Justin Greenwood/ Joanna Dreger, The Transparency Register, in: Interest Groups and Advocacy, 2. Jg. (2013), H. 2, S. $139-162$.

60 Vgl. Burson-Marsteller Europe, A Guide to Effective Lobbying in Europe: The Views of PolicyMakers, Brüssel 2013, http://lobbyingsurvey.burson-marsteller.eu/wp-content/uploads/2013/05/ european_lobbying_survey_2013.pdf(Abruf am 3. September 2014), S. 64.

61 Vgl. Alter-EU, A Year of Broken Promises: Big Business Still Put in Charge of EU Expert Groups, Despite Commitment to Reform, Brüssel 2013, http://www.alter-eu.org/sites/default/files/documents/Broken_Promises_web.pdf (Abruf am 3. September 2014).

$62 \mathrm{Vgl}$. Heike Klüver, Biasing Politics? Interest Group Participation in EU Policy-making, in: West European Politics, 35. Jg. (2012), H. 5, S. $1114-1133$.

63 Vgl. European Commission, The EU Anti-Corruption Report (COM 2014/38), Brüssel 2014. 
Umfragen unter Bürgern und Unternehmen in 27 EU-Ländern (ohne Kroatien). ${ }^{64}$ Laut der befragten Bürger besteht Korruption, durch die Kommission definiert als „Machtmissbrauch für privaten Gewinn“, überall, jedoch am wenigsten in den nördlichen und am meisten in den östlichen und südlichen Ländern. Laut den Unternehmen gilt dies auch bei öffentlichen Ausschreibungen, die insbesondere eine EU-Kompetenz sind. Deutschland belegt unter den besseren Ländern insgesamt die sechste Position. Die Korruptionspraktiken finden aus Sicht der Befragten meistens zwischen Parteien, Behörden und Unternehmen sowie auch innerhalb dieser Akteure statt. Eine hohe Korruptionsperzeption der Bürger korreliert mit Misstrauen in ihre jeweiligen Institutionen, was das hohe Vertrauen der mittel- und osteuropäischen Staaten in das EP erklären würde. ${ }^{65}$ Nachdem die Unternehmen besser etabliert sind, respektieren sie mehr die Grenzen der Beeinflussung. ${ }^{66}$ Auf EU-Ebene gibt es keine vergleichbaren Daten, nur eine erste Umfrage unter fast 50 leitenden Amtspersonen aus zehn EU-Institutionen, ob „die EU, gemessen nach Prozeduren und Prakti-

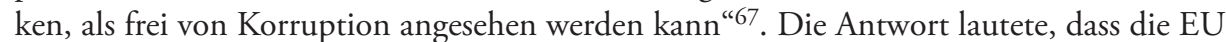
nicht frei von Korruptionsrisiken ist. Wie dem auch sei, die Medien berichten jährlich viel seltener über Skandale in der EU als in den „besseren“ Ländern. Brüssel sieht aus wie ein Dorf mit hoher Sozialkontrolle.

Diese Befunde, Hinweise und Daten bedeuten nicht, dass die Demokratievorstellungen auf der EU-Ebene perfekt verwirklicht sind, sondern nur, dass auf dieser Ebene Akteure und Faktoren, die den Demokratiegehalt verstärken, besonders wirksam sind. Dass in der EU die Beeinflussung durch Interessengruppen heute einen „besseren Demokratiegehalt“ als in der Vergangenheit und in den EU-Mitgliedstaaten zeigt, lässt sich nicht einfach erklären. Auch in vielen Ländern gibt es Transparenzinitiativen, Regelungen, Mediendebatten usw., die aber offenbar noch nicht genügend effektiv sind. Unterschiede zwischen der EUEbene und den Ländern könnten erklärende Hypothesen liefern. Sichtbar sind unter anderem die auf EU-Ebene größere Zahl von Interessengruppen, der stärkere Wettbewerb, der offenere Zugang, die stärkere Sozialkontrolle und die lebendigere Praxismoral, die teils zusammenhängen. Hinzu kommen eher versteckte Unterschiede, beispielsweise die auf der EU-Ebene selbstkorrigierenden Mechanismen, die hier fast abwesende Parteipolitik, der kleinere und multinationalere Beamtenapparat und die Verwandlung von altem Lobbyismus in moderneres Public Affairs Management. Solche Hypothesen benötigen wissenschaftliche Untersuchungen. Ausgehend von der heutigen Lage der doppelt vergleichenden Forschung (Parteien und Interessengruppen auf zwei Ebenen), müssen diese Untersuchungen vor allem empirisch-induktiv angesetzt werden.

64 Vgl. dies., Corruption, Special Eurobarometer 397, Brüssel 2014, http://ec.europa.eu/public_ opinion/archives/ebs/ebs_397_en.pdf; dies., Business Attitudes towards Corruption in the EU, Flash Eurobarometer 374, Brüssel 2014, http://ec.europa.eu/public_opinion/flash/fl_374_en.pdf (jeweils Abruf am 3. September 2014).

65 Vgl. Christine Arnold / Eliyahu Sapir / Galina Zapryanova, Trust in the EU Institutions, in: European Integration online Papers, 16. Jg. (2012), H. 2, S. 1 - 39; Jordi Muňoz / Mariano Torcal / Eduard Bonet, a.a.O. (Fn. 55).

66 Vgl. Morten Bennedsen / Sven Feldman / David Dreyer Lassen, Lobbying and Bribes: A Surveybased Analysis of the Demand for Influence and Corruption, in: CESifo Working Paper: Public Finance Nr. 3496, 2011, http://www.econstor.eu/bitstream/10419/46525/1/662659597.pdf (Abruf am 3. September 2014).

67 Transparency International EU, The European Union Integrity System, Brüssel 2014. 


\section{Resümee und Ausblick: Wie können die Parteien ihren Rückstand in der EU aufholen?}

Auf der Nationalebene der meisten EU-Länder haben die Parteien starke Machtpositionen im Gegensatz zu den Interessengruppen, die ihren Mangel daran durch die Beeinflussung von Politikern, Behörden und Stakeholdern zu kompensieren versuchen. Beide Hauptformen gesellschaftlicher Organisation haben keine fest etablierten Beziehungen zu den Bürgern in ihren Rollen als Mitglieder und Wähler beziehungsweise Interessenten, jedoch sind die der Interessengruppen viel zahlreicher und vielgestaltiger, da die Bürger bedeutend mehr (spezifische) Interessen haben als ihre (allgemeine) Parteipräferenz.

Auf der EU-Ebene verflüchtigen sich die nationalen Parteien. Im EP ist jede Partei eine sehr kleine Minorität, die sich wie eine Interessegruppe verhalten muss. Erstens muss sie dies innerhalb einer politischen Gruppe und zweitens im Plenum tun, bevor sie sich an den Machtpositionen des Parlaments bei der EU-Gesetzgebung beteiligen kann. Auch außerhalb dieser EP-Innenwelt sind die Parteien und die Gruppen sowie das EP angewiesen auf Beeinflussung durch Interessenpolitik. Die Interessengruppen verstehen auf nationaler Ebene das Spiel der Beeinflussung schon besser, setzen dieses fort in der EU, gruppieren sich dort um zu länder- und oft zu sektorenübergreifenden EuroFeds und/oder Netzwerken, erhalten durch diese starke Positionen beispielsweise in den Expertengruppen der Kommission und zeigen durch ihren Interessenhandel und -wandel - einerseits untereinander und andererseits mit den Institutionen -, dass sie viele Entscheidungsprozesse beeinflussen können. Bislang sind die Parteien die Verlierer und die Interessengruppen die Gewinner der Europäisierung ihres Landes.

Auch die vielen verschiedenen Demokratievorstellungen der Mitgliedstaaten sind inzwischen auf EU-Ebene in einem Europäisierungsprozess begriffen, der ein neues Demokratieamalgam erzeugt. In allen Ländern sind die Parteien gesetzlich stark in der Staatsordnung positioniert, jedoch nicht auf EU-Ebene. Solange die Wahlen für das EP in etwa 200 verschiedenen nationalen Parteien resultieren, wird diese Situation sich kaum ändern. Viele nationale Parlamente versuchen zur Zeit, durch die Subsidiaritätsprozedur und auf andere Weisen, ihre Parteien national in eine Mehrheitsposition über EU-Fragen zu bringen, die dann mit vielen anderen nationalen Parlamenten verhandelt werden muss, um die Kommission beeinflussen zu können. Bisher haben diese Versuche kaum nennenswerten Erfolg gehabt. Der neue Fokus der Fraktionen auf nationale Verfahren hilft ihnen kaum, in Brüssel ihre schwache Präsenz, Etablierung und Professionalität zur Beeinflussung der europäischen Politik zu verbessern. Auf diese Weise übergehen die nationalen Parlamente sogar das EP, konkurrieren mit ihm und stimulieren ihre nationalen Oppositionsparteien, im EP als Gegenspieler aufzutreten. Neben den nationalen Parteien bleiben die nationalen Parlamente vorläufig, trotz ihrer oft widersprechenden Rhetorik, in den Augen der Bürger die Verlierer der EU-Integration.

Die nationalen Parlamente und ihre Parteien benehmen sich nicht wie eine moderne Interessengruppe. Anstatt sich auf die EU-Prozesse zu konzentrieren, um die Entscheidungsfindung zu beeinflussen, sind sie fixiert auf Verfahren, entweder mit ihrer Regierung, die meistens im Rat enden, oder mit der Kommission (die Subsidiaritätsprozedur), was wenig Erfolg verspricht. Es gibt keinen guten Grund, warum in der EU die Parlamente nicht Einflusserfolge wie Großkonzerne erzielen können, und die Parteien wie die KMU. Ihr neuer Aktivismus bietet nun einen guten Ansatz. Wenn sie ihren Rückstand auf Interessengruppen einholen wollen, müssen sie ihre Präsenz, Etablierung und Professionalität in 
der EU verbessern. Dazu brauchen auch sie weitergehende Ambitionen, Intelligenz und Umsicht. Der Erwerb dieser drei sachlich-politischen Qualitäten des Public Affairs Managements wird an Parlamente, Parteien und Politiker hohe Anforderungen stellen. Beeinflussung ist vor allem ein Fachberuf, für den die Berufung allein nicht ausreicht.

Nationale Parlamente, Parteien und Parteipolitiker könnten am besten pragmatisch damit anfangen, drei verfügbare Stakeholder nicht nur gelegentlich, sondern strukturell häufiger und besser zu nutzen:

(1) Die interparlamentarische COSAC (der Zusammenschluss der nationalen EU-Ausschüsse, Conférence des Organisations spécialisées aux affaires communautaires) wird bis jetzt vor allem als Informationsplattform (IPEX) genutzt und selten als eine EuroFed, die auch eine gemeinsame, weitere Agenda zur Beeinflussung der EU darstellen könnte. Dieses Versäumnis könnte und müsste schnell beendet werden.

(2) Die Fraktionen und Parteipolitiker in den nationalen Parlamenten benutzen noch wenig ihre befreundeten Interessengruppen, die in der EU aktiv, in Brüssel gut etabliert und bei der Kommission stark in den Expertengruppen positioniert sind. Eine positive Ausnahme zeigten zum Beispiel die Grünen in einigen Parlamenten (und im EP), die oft mit der Umweltbewegung direkt zusammenarbeiten (um nicht zu sagen, sich inszenieren lassen). Auch eine aktivere Benutzung der EP-Intergruppen kann dazu beitragen, dass sich die Parteien mit der EU-Entscheidungsfindung schneller und besser vertraut machen.

(3) Die Parteien in den Mitgliedstaaten haben noch immer schwache Beziehungen zu ihren Bürgern, die seit 2007 durch den Lissabon-Vertrag in EU-Fragen relevantere Stakeholder geworden sind. Um mit ihnen zu kommunizieren, sind die Parteien, so lange die neuen Medien wenig Tiefgang und Reichweite bieten, stark von den alten Massenmedien abhängig. Diese publizieren EU-Berichte der Parteien jedoch meist nur, wenn sich diese polarisierend darstellen lassen, was die Regierungsparteien und ihre Mitglieder häufig abschreckt. ${ }^{68}$ Dabei behindert das Misstrauen vieler Bürger in die Parteien eine gute Kommunikation. Auch deshalb müssen die Parteien ihre Interessengruppen mehr nutzen, da diese oft mehr Vertrauen der Bürger genießen und folglich einen effektiveren Kommunikations(um)weg bieten.

Die Vorteile solch einer pragmatischen Arbeitsweise sind, erstens, dass eine als transnationale EuroFed funktionierende COSAC durch ihre strukturell gemeinsame Willensbildung extern mehr Beeinflussungsmöglichkeiten besonders bei der Kommission und den Stakeholdern eröffnet; zweitens, dass viele Interessengruppen aus Eigennutz den Parteien und Politikern (sowie ihren Mitarbeitern) zweckdienliche Informationen und eine „kritische“ Menge an Unterstützung bereitstellen $\left(\mathrm{MI}^{2}\right)$, mithin effektivere und effizientere Beeinflussung ermöglichen und sie spielend über die entstehenden flexiblen Allianzen zu Public Affairs Management in der EU erziehen; und drittens, dass die Bürger öfter wahrnehmen, dass ihr Parlament, ihre Parteien und ihre Politiker auf dem Weg sind, sich in der EU aktiver, etablierter und professioneller zu verhalten. Wer im Rückstand ist, muss nicht frustriert, sondern gescheit sein und sich durch Hebel, Kupplungen und Multiplikatoren beschleunigen.

68 Vgl. Katrin Auel / Tapio Raunio (Hrsg.), Special Issue: Connecting with the Electorate? Parliamentary Communication in EU Affairs, in: Journal of Legislative Studies, 20. Jg. (2014), H. 1, S. 1 - 138; Hajo Boomgaarden / Claes de Vreese, Explaining Variation in News Coverage of the EU, in: European Journal of Political research, 52. Jg. (2013), H. 3, S. 403 - 421; Erik Eriksen 1 John Fossum, Rethinking Democracy and the EU, London 2012. 